\title{
Quantum oscillations from networked topological interfaces in a Weyl semimetal
}

\author{
I-Lin Liu (iD ${ }^{1,2,3 凶}$, Colin Heikes (iD) ${ }^{1,4}$, Taner Yildirim ${ }^{1}$, Chris Eckberg ${ }^{2}$, Tristin Metz ${ }^{2}$, Hyunsoo Kim (iD) ${ }^{2}$, Sheng Ran ${ }^{1,2,3,5}$, \\ William D. Ratcliff $\mathbb{D}^{1}$, Johnpierre Paglione $\mathbb{D i D}^{2}$ and Nicholas P. Butch $\mathbb{1}^{1,2 凶}$
}

Layered transition metal chalcogenides are promising hosts of electronic Weyl nodes and topological superconductivity. MoTe ${ }_{2}$ is a striking example that harbors both noncentrosymmetric $T_{d}$ and centrosymmetric $T^{\prime}$ phases, both of which have been identified as topologically nontrivial. Applied pressure tunes the structural transition separating these phases to zero temperature, stabilizing a mixed $T_{d}-T^{\prime}$ matrix that entails a network of interfaces between the two nontrivial topological phases. Here, we show that this critical pressure range is characterized by distinct coherent quantum oscillations, indicating that the difference in topology between topologically nonvtrivial $T_{d}$ and $T^{\prime}$ phases gives rise to an emergent electronic structure: a network of topological interfaces. A rare combination of topologically nontrivial electronic structures and locked-in transformation barriers leads to this counterintuitive situation, wherein quantum oscillations can be observed in a structurally inhomogeneous material. These results further open the possibility of stabilizing multiple topological phases coexisting with superconductivity.

npj Quantum Materials (2020)5:62; https://doi.org/10.1038/s41535-020-00264-8

\section{INTRODUCTION}

Topologically protected electronic states at material interfaces are attractive because they cannot be destroyed by many types of perturbations ${ }^{1,2}$. An especially fruitful host of such exotic states is $\mathrm{MoTe}_{2}$, which has had both its bulk orthorhombic $\mathrm{T}_{\mathrm{d}}$ phase, and hole-doped monolayer specimens identified as possible topological superconductors ${ }^{3-5}$. In addition, a topological superconducting phase was recently discovered in sulfur-substituted samples, with $S_{+-}$-wave pairing ${ }^{6}$. MoTe ${ }_{2}$ also features topologically nontrivial normal states: the $T_{d}$ phase has been identified as a type II Weyl semimetal ${ }^{7-10}$, whereas the monoclinic $\mathrm{T}^{\prime}$ phase is predicted to be a higher-order topological material ${ }^{11}$; the latter is also found to describe the $T_{d}$ phase in some calculations ${ }^{11}$. In this work, we demonstrate via quantum oscillations and neutron scattering measurements, and first-principles calculations, how pressure drives $\mathrm{MoTe}_{2}$ between the $\mathrm{T}_{\mathrm{d}}$ and $\mathrm{T}^{\prime}$ phases, through an intermediate mixed-phase region. The mixed-phase region gives rise to a network of topological interface states that yield quantum oscillations that survive despite the strong structural disorder.

\section{RESULTS}

Orthorhombic $\mathrm{T}_{\mathrm{d}}-\mathrm{MoTe}_{2}$

The first-order structural transition separating the $T^{\prime}$ and $T_{d}$ phases in $\mathrm{MoTe}_{2}$ has a distinct pressure dependence (Fig. 1a). At ambient pressure, the inversion-symmetric $\mathrm{T}^{\prime}$ phase is stable at room temperature, only transforming into the noncentrosymmetric $T_{d}$ phase when cooled below roughly $250 \mathrm{~K}^{3,12}$. Neutron diffraction allows the determination of the relative volume fraction of these phases under different conditions ${ }^{12}$. As pressure increases, the transition temperature decreases. At pressures higher than $0.8 \mathrm{GPa}$, a completely different phenomenon emerges, where a roughly balanced mixture of the $T^{\prime}$ and $T_{d}$ phases stabilizes over an appreciable temperature range, and crucially, extends to the lowest measured temperatures. The existence of this frozen mixed-phase region is stabilized by the lack of sufficient entropy at these suppressed temperatures for atoms to move to their lowest-energy configuration, implying that there is a dominant extrinsic transformation energy barrier between two energetically nearly degenerate structures ${ }^{12}$.

The basic components underlying the Weyl semimetallic state of the low-pressure, low-temperature $T_{d}$ phase are a large hole pocket centered on the Brillouin zone and two neighboring electron pockets along the $\Gamma-X$ direction ${ }^{8-10,13}$. The hole pocket is observed in angle-resolved photoemission spectroscopy (ARPES) ${ }^{8-10}$, but is not apparent in SdH measurements ${ }^{14}$. Prominent quantum oscillations observed in the $T_{d}$ phase arise from orbits associated with the electron pocket ${ }^{14}$. Figure $2 \mathrm{a}, \mathrm{b}$ shows magnetoresistance and $\mathrm{SdH}$ oscillations at ambient pressure, in which these are clearly seen. As the fast Fourier transform (FFT) explicitly shows (Fig. 2c), the beating seen in Fig. $2 \mathrm{~b}$ is due to two similar frequencies, $F_{\alpha}=240.5 \mathrm{~T}$ and $F_{\beta}=258 \mathrm{~T}$, the result of symmetry-allowed spin-dorbit splitting. First-principles calculations identify these frequencies with the larger extremal $\mathbf{k}_{\mathrm{z}}=0$ cross sections of the electron pocket.

Modeling of the $\mathrm{SdH}$ oscillations yields a remarkably good fit (Fig. 2d) to the experimental $\mathrm{SdH}$ by the Bumps program ${ }^{15}$ (Supplementary Figs. 15-23, Supplementary Table 5, Supplementary Notes 9 and 10). Notably, all of the oscillations feature a $\pi$ Berry's phase ${ }^{16}$, consistent with a Weyl topology (Supplementary Figs. 3-7, 14, and Supplementary Note 2). The effective band masses are light and slightly less than previously reported ${ }^{14,17}$, as shown in Fig. 2e. As a function of pressure, the electron pockets increase modestly in size due to lattice compression, but the nontrivial phase shift is maintained throughout the $T_{d}$ phase (Supplementary Tables 1-4). This trend is consistent with firstprinciples calculations as shown in Fig. $3 a$ and $b$, which indicates the persistence of Weyl nodes up to 1.4 GPa (Supplementary Fig. 6 and Supplementary Note 2).

\footnotetext{
${ }^{1}$ NIST Center for Neutron Research, NIST, Gaithersburg, MD 20899, USA. ${ }^{2}$ Center for Nanophysics and Advanced Materials, Department of Physics, University of Maryland, College Park, MD 20742, USA. ${ }^{3}$ Department of Materials Science and Engineering, University of Maryland, College Park, MD 20742, USA. ${ }^{4}$ Joint Quantum Institute, NIST and University of Maryland, College Park, MD 20742, USA. ${ }^{5}$ Department of Physics, Washington University in St. Louis, St. Louis, MO 63130, USA. ${ }^{\mathrm{e}} \mathrm{email}$ : ilin610@umd.edu; nbutch@umd.edu
} 

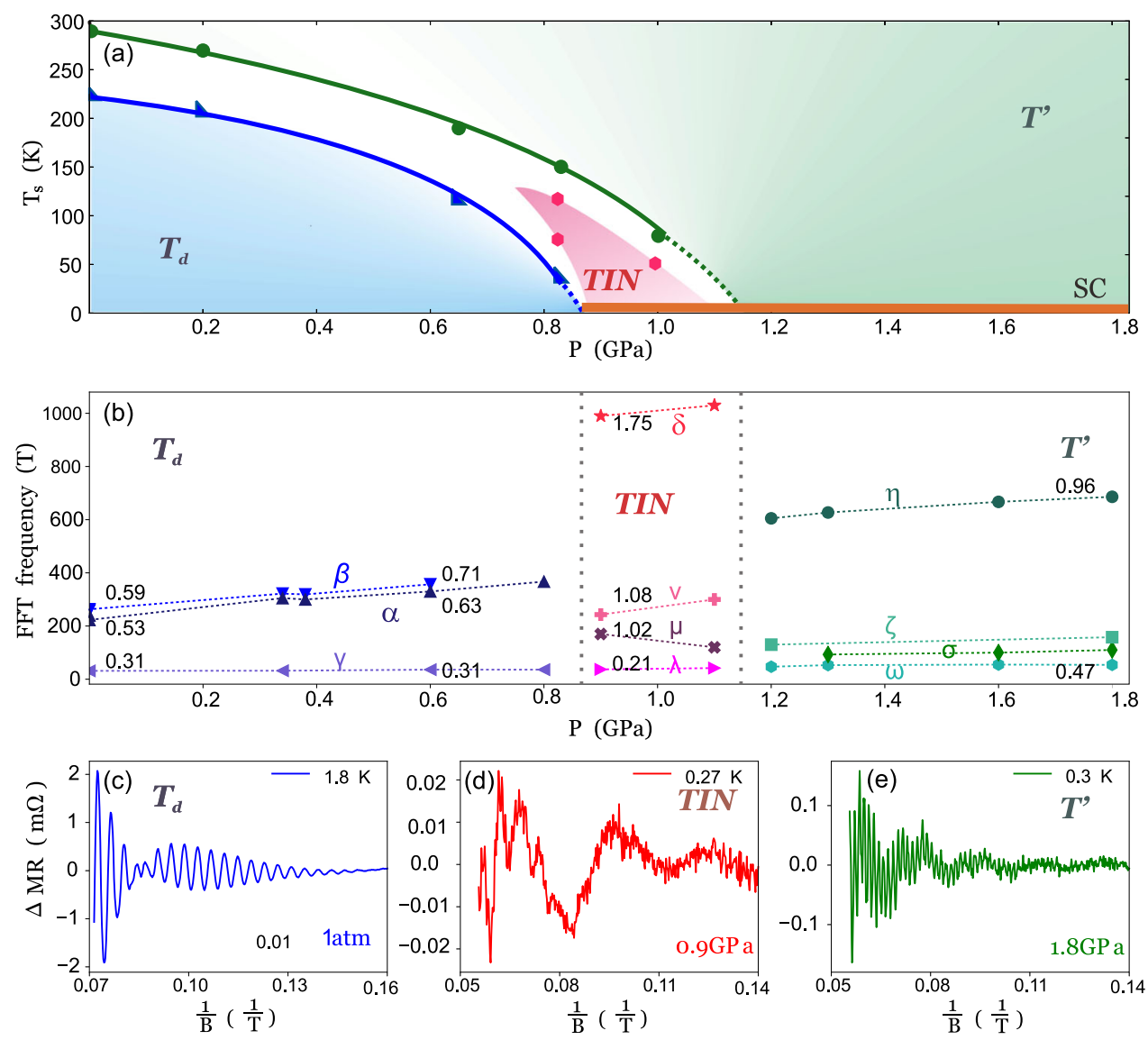

Fig. 1 Pressure-temperature phase diagram of $\mathrm{MoTe}_{2}$. a Pressure-temperature phase diagram of MoTe $\mathrm{T}_{2}$. The green and blue symbols delineate the extent of $100 \%$ volume fraction of $\mathrm{T}^{\prime}$ and $\mathrm{T}_{\mathrm{d}}$ phases, respectively, determined through neutron scattering. The onset of full volume superconductivity coincides with the end of the $\mathrm{T}_{d}$ phase at $0.8 \mathrm{GPa}{ }^{12}$, where a topological interface network (TIN) is observed. b Pressure dependence of the quantum oscillation frequency, with different branches labeled by Greek letters. The numbers correspond to the effective mass, which changes slightly with pressure. The quantum oscillations with the strongest pressure dependence, $a$ and $\beta$ in the $T_{d}$ phase, and $\eta$ in the T' phase, correspond to extremal orbits on the large electron pockets. In the TIN region, these disappear and are replaced by a completely distinct set of oscillations arising from topological interface states. Representative quantum oscillations (c) at ambient pressure (Weyl semimetal in $\mathrm{T}_{\mathrm{d}}$ ), d $0.9 \mathrm{GPa}(\mathrm{TIN})$, and e $1.8 \mathrm{GPa}$ (higher-order topology in $\mathrm{T}^{\prime}$ ). Clear changes in the quantum oscillations reflect significant changes in the electronic structure.

\section{Monoclinic $\mathrm{T}^{\prime}-\mathrm{MoTe}_{2}$}

In the $T^{\prime}$ phase at pressures of $1.2 \mathrm{GPa}$ and greater, one main frequency $F_{\eta}$ replaces the two frequencies $F_{\alpha}$ and $F_{\beta}$. The Fermi surface in the $T^{\prime}$ phase closely resembles that of the $T_{d}$ phase, with the exception that the centrosymmetry of the $\mathrm{T}^{\prime}$ phase nullifies the spin-orbit splitting of the bands that contribute to this electron pocket (Fig. 3d, e). This similarity leads to a common pressure dependence of the measured electron pocket frequency in both phases, consistent with the pressure evolution of the calculated Fermi surface (Fig. 3c, Supplementary Fig. 8, and Supplementary Tables 1-4). The main feature not captured by the calculations is a pronounced discontinuity in band structure between $T_{d}$ and $T^{\prime}$ phases due to the discontinuous $\mathbf{c}$ axis dilation at the structural transition, which is not modeled (Fig. 3c). Unexpectedly, our SdH oscillations analysis shows that a $\pi$ Berry's phase also exists in the centrosymmetric $T^{\prime}$ phase (Supplementary Table S6). After an ARPES study identified surface states in this phase $^{13}$, it was identified theoretically with an unusual type of nontrivial topological state ${ }^{11}$. Our results are consistent with this prediction, and further have the exciting implication that the high-pressure superconductivity in the $\mathrm{T}^{\prime}$ phase may be inherently topologically nontrivial, as suggested by $\mu \mathrm{SR}$ measurements at these pressures ${ }^{18}$.
Natural topological interface network (TIN)

A structural mixed region exists over a range of pressures and temperatures in between the bulk $T_{d}$ and $T^{\prime}$ phases (Fig. 1a). It consists of an approximately balanced partial volume fraction of $\mathrm{T}_{d}$ and $\mathrm{T}^{\prime}$. We emphasize that no other structural phases or ordered superstructures are apparent from neutron diffraction measurements (Supplementary Figs. 1 and 2). It would, therefore, be expected that any measured $\mathrm{SdH}$ oscillations in the mixed region would consist of a superposition of $T_{d}$ and $T^{\prime}$ signals, but we do not observe oscillations from either phase. We conclude that the mixed region is sufficiently disordered that $\mathrm{SdH}$ oscillations from both the $\mathrm{T}_{d}$ and $\mathrm{T}^{\prime}$ phases are suppressed due to increased electron scattering.

In light of this, it is completely unexpected that a distinct set of $\mathrm{SdH}$ oscillations appears (Fig. 1b, d). This is a robust effect; the oscillations in the mixed phase always appear on both increasing and decreasing applied pressures through the critical range, over multiple cycles, confirming their intrinsic nature. A different band structure in the mixed region is inferred from the presence of frequencies corresponding to changed Fermi surfaces, and a change in effective mass and much weaker oscillation amplitude relative to bulk $T_{d}$ and $T^{\prime}$, as shown in Fig. 4a-f. These quantum oscillations reflect features typical of topologically protected states, namely, persistence in the presence of strong disorder, as 

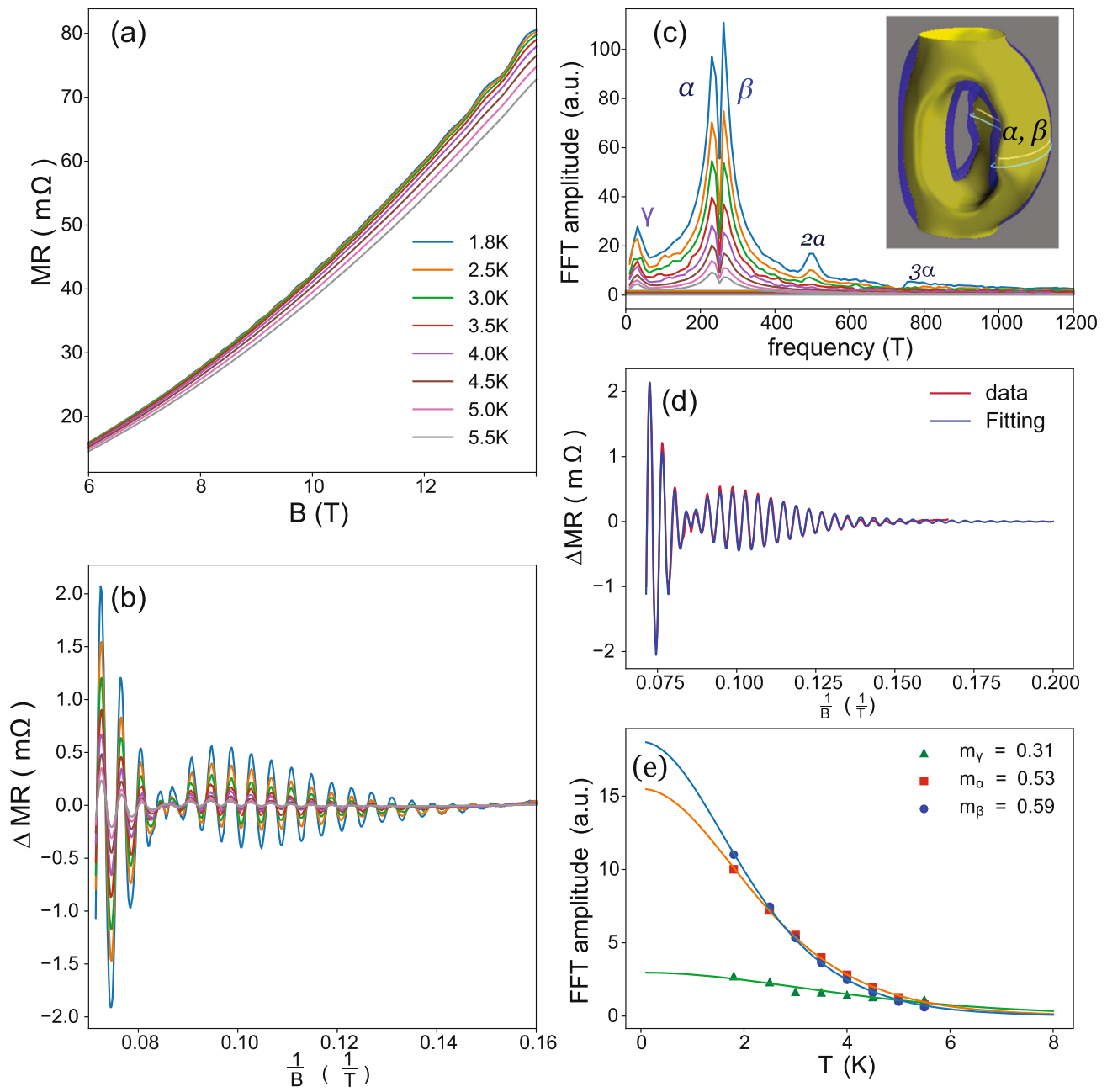

Fig. 2 Quantum oscillations in the magnetoresistance in $\mathrm{MoTe}_{2}$ at ambient pressure. a The longitudinal $M R$ of the bulk $\mathrm{T}_{d}$-MoTe $\mathrm{M}_{2}$ measured at ambient pressure with a magnetic field parallel to the $\mathbf{c}$ axis. b The corresponding $\mathrm{SdH}$ oscillations after subtraction of a second-order polynomial background. c The fast Fourier transform (FFT) spectra show three Fermi surfaces with oscillation frequencies at $F_{\gamma}=32.5 \mathrm{~T}$, $F_{\alpha}=$ $240.5 \mathrm{~T}$, and $F_{\beta}=258 \mathrm{~T}$. The inset shows the $\alpha$ and $\beta$ orbits on the calculated electron pocket. $\mathbf{d}$ Fit to the SdH oscillation at $1.8 \mathrm{~K}$ yield nontrivial Berry's phases $\phi_{\gamma}=\pi, \phi_{\alpha}=0.88 \pi$, and $\phi_{\beta}=0.88 \pi$. Details of the fit are discussed in the Supplementary Information. e The effective masses of the carriers are obtained through temperature-dependent Lifshitz-Kosevich fits.

well as $\pi$ Berry's phases (Supplementary Tables 5 and 6). Because there are no additional structural phases in the mixed region, only $\mathrm{T}_{\mathrm{d}}$ and $\mathrm{T}^{\prime}$, these topological states must be surface states of the bulk phases, which in this case, exist at their interfaces. Thus, the multiple natural interfaces stabilized in $\mathrm{MoTe}_{2}$ by the first-order nature of the structural transition serve as the foundation for a completely different type of electronic system in $\mathrm{MoTe}_{2}$ : a natural topological interface network (TIN).

In the TIN, due to the layered structure of both $T_{d}$ and $T^{\prime}$ phases, the ab plane is preserved, and the largest grain boundaries fall along the $\mathbf{a b}$ plane, which is the orientation probed by the $\mathrm{SdH}$ measurements (Fig. $4 \mathrm{~g}$ ). In $\mathrm{MoTe}_{2}$, a naturally generated heterostructure provides an interesting demonstration of topological transport protection. The lateral dimensions of the interfaces are the same as those of the bulk grains, based upon which one naively expects similar damping of the $\mathrm{SdH}$ oscillations from the interfaces. The absence of quantum oscillations from bulk $T_{d}$ and $T^{\prime}$ phases illustrates that the scattering from the TIN microstructure is significant for the bulk bands. Yet the clear $\mathrm{SdH}$ oscillations from the interfaces prove that the interfacial states have lower scattering than the bulk, and are a sign of their topologically nontrivial nature. In other words, the interfacial signal has been amplified by suppressing the bulk SdH oscillations through grain boundary scattering, and increasing the interface volume.

Density-functional theory (DFT) calculation of $T_{d}-T^{\prime}$ slab model First-principles calculations offer additional insight into the electronic structure of the TIN and its stability. We investigated a variety of possible mixed phases that might describe the mixed region, including $T_{d}$-like phases with different $M_{0} T_{2}$ stacking orders, periodic superstructures, and finite slabs of $T_{d}$ and $T^{\prime}$ phases and their interfaces as shown in Fig. $5 \mathrm{a}$ and $\mathrm{b}$ (detailed calculations are discussed in the Supplementary Figs. 9-13 and Supplementary Notes 4-6). Calculations demonstrate that the only model consistent with the experimentally determined highfrequency oscillations of $\sim 1 \mathrm{kT}$ (Fig. $4 \mathrm{~d}$ ) is a system consisting of only $T_{d}-T^{\prime}$ interfaces, the TIN (Fig. $5 d$ ).

The Fermi-surface pockets associated with the interface are identified in two different but complementary calculations. Consider a periodic superstructure of $T_{d}$ and $T^{\prime}$ phases, as shown in Fig. 5a. Surprisingly, the $T_{d}-T^{\prime}$ interface has lower energy if the $\mathrm{MoTe}_{2}$ layers at the interface have the same type of Te distortion, labeled as $L$ or $R$, compared to the L-R-L-R-type stacking order found in bulk $T_{d}$ and $T^{\prime}$ phases. Since $L$ - or R-type planes cannot be 

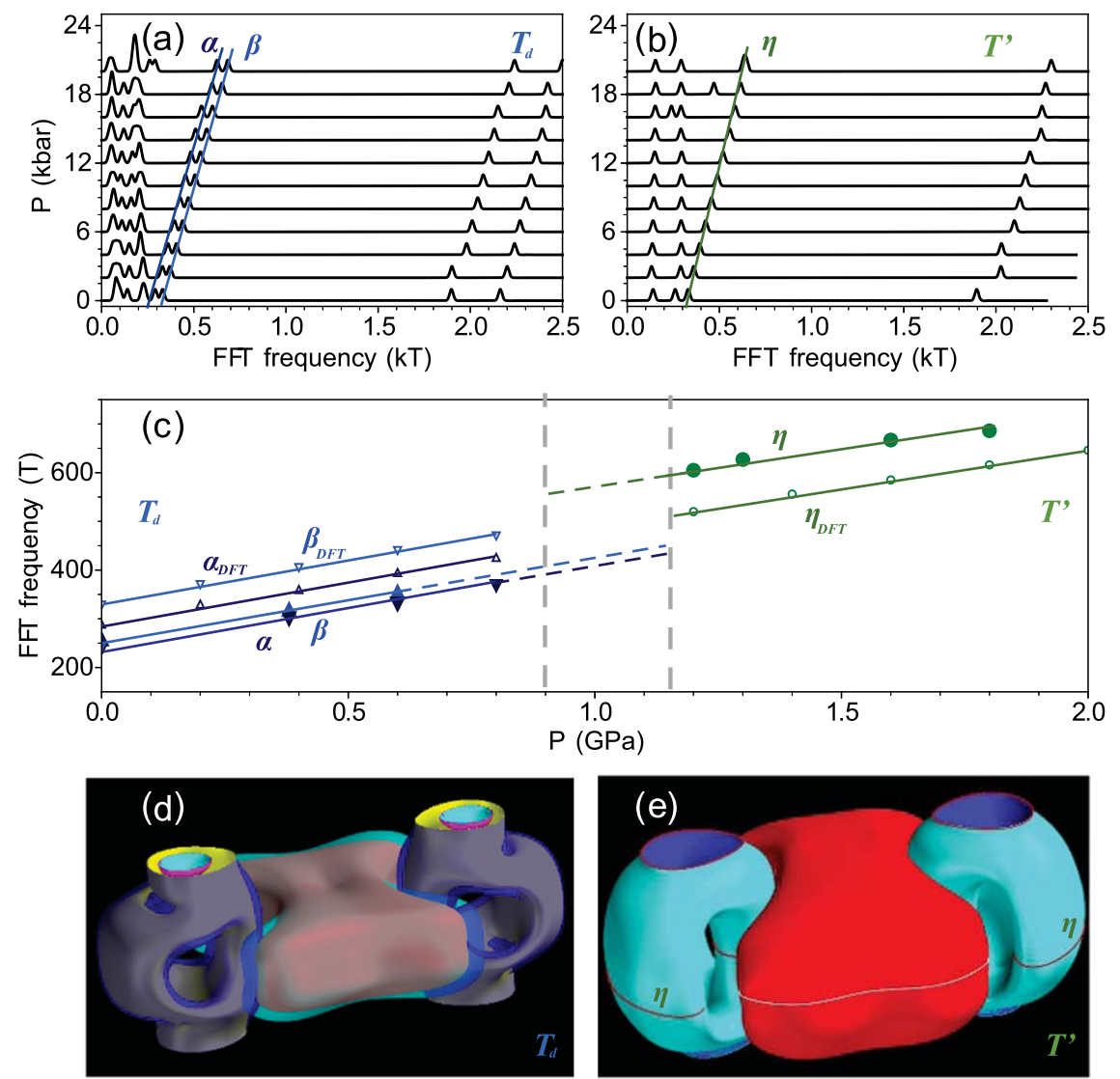

Fig. 3 Comparison between the calculated and experimental quantum oscillation frequencies for $\mathbf{T}_{\mathbf{d}}$ and $\mathbf{T}^{\prime}$ phases. a, $\mathbf{b}$ Calculated inplane quantum oscillation frequencies for the $T_{d}$ and $\mathrm{T}^{\prime}$ phases. The $\mathrm{SdH}$ oscillation frequencies arising from the electron pockets increase with the same pressure dependence in both phases. c A comparison between calculated and measured frequencies shows excellent quantitative agreement. The discontinuity in measured values is due to the jump in c axis dilation between phases. $\mathbf{d}$, e Calculated bulk Fermi surfaces of the $T_{d}$ and $T^{\prime}$ phases.

converted to each other by simply sliding the planes, this sort of interface presents a large energy barrier to the removal of stacking faults. The fact that this LL-type interface has lower energy than the interface with L-R stacking explains the stability of the mixed intermediate phase. Comparing the Fermi surfaces of the pure $T_{d}$ and $T^{\prime}$ phases with the $T_{d}-T^{\prime}$ superstructure yields distinct Fermisurface pockets with quantum oscillation orbits of $0.16 \mathrm{kT}$ and $1.0 \mathrm{kT}$ (bottom panel of Fig. $5 \mathrm{~d}$ ) in excellent agreement with our measurements.

Even though the periodic superstructure calculations of the $T_{d}-T^{\prime}$ interface are consistent with the TIN model, the interface pockets have some dispersion due to interactions between the periodic images of the interface. To eliminate this effect, consider two slabs of $T_{d}$ and $T^{\prime}$ initially separated by about $9.5 \AA$ (Fig.5b). These two slabs are brought together to form the $T_{d}-T^{\prime}$ interface in a supercell where the combined $T_{d}-T^{\prime}$ slab is separated by $\sim 16.3 \AA$, large enough to avoid any image interactions (Fig. $5 \mathrm{~b}$, bottom). Comparing the Fermi surfaces of the isolated slabs (Fig. 5b) and combined slabs with the interface (shown in Fig. 5c), yields Fermi-surface pockets associated with the interface (Fig. 5d, top).

The agreement is excellent between the surface states from the periodic and finite slab calculations. These 2D quantum oscillation orbits are in impressive agreement with the experimental results (middle panel of Fig. 5d). Thus, the slab calculations directly link the observed high-frequency oscillation in the mixed region to interface states, as a TIN would produce.

\section{DISCUSSION}

Essential to any topological surface state is the meeting of two bulk phases with different topological invariants. A typical example is that of a Weyl semimetal interfaced with a topologically trivial vacuum, on whose boundary Fermi arcs are observed, as in $\mathrm{T}_{\mathrm{d}}-\mathrm{MoTe}_{2}{ }^{8}$. In contrast, the second phase of the TIN is the topologically nontrivial $\mathrm{T}^{\prime}$ phase, which forms a large number of $T_{d} / T^{\prime}$ interfaces due to the structural disorder. Then, for surface states to exist at these interfaces, there must be a change in topology between $T_{d}$ and $T^{\prime}$ phase, as expected for phases of Weyl and higher-order topological states. Although such interfaces have until now not received much theoretical attention, recent scanning tunneling microscopy measurements on $\mathrm{MoTe}_{2}$ have suggested the existence of conducting hinge states in the $\mathrm{T}^{\prime} /$ $\mathrm{T}_{\mathrm{d}}$ domain wall at ambient pressure ${ }^{19}$. Our results did not directly observe 1D-hinge state, but cannot completely exclude the possibility of $1 \mathrm{D}$ paths. Our measurements and calculations show that 2D topological interfaces are also possible. Our discovery suggests that topological interfaces in 3D materials are a rich area for future exploration and exploitation.

Finally, we briefly discuss the relationship between the TIN and superconductivity in $\mathrm{MoTe}_{2}$. Both the superconducting volume fraction and $T_{c}$ increase dramatically at the same pressure at which the TIN is stabilized (Fig. 1a). Taking into further consideration the 2D nature of superconductivity, based on our previous calculations $^{12}$ and recent upper critical field measurements under pressure 20 a strong case is made that superconductivity is topologically nontrivial, consistent with previous claims from 

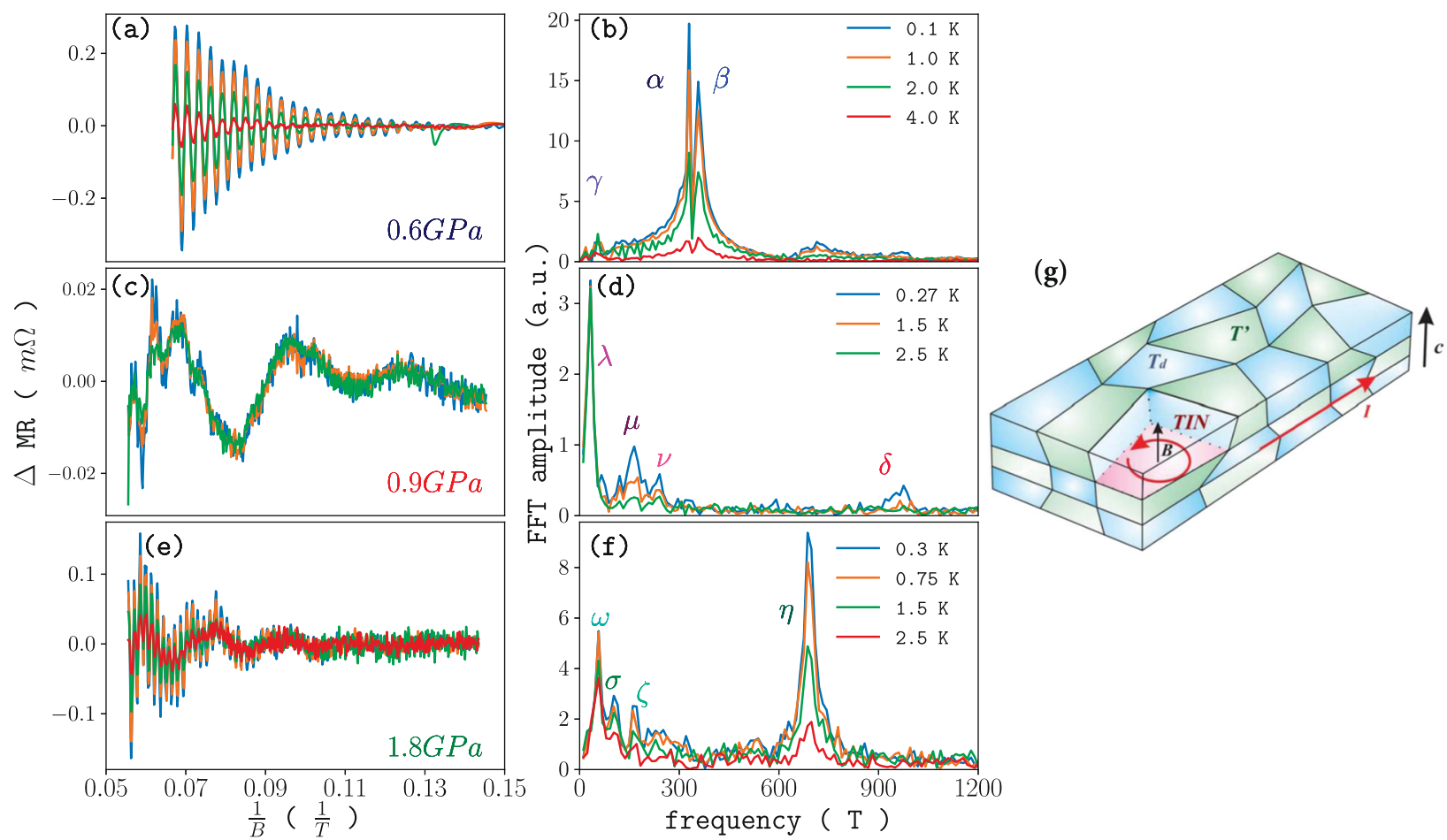

Fig. 4 Quantum oscillations in the magnetoresistance in $\mathrm{MoTe}_{2}$ under pressure. Temperature dependence of the $\mathrm{SdH}$ oscillations of MoTe 2 measured at (a) $0.6 \mathrm{GPa}$ in the $\mathrm{T}_{d}$ phase, (c) $0.9 \mathrm{GPa}$ in the topological interface network (TIN), and (e) $1.8 \mathrm{GPa}$ in the T' phase. Note the large changes in oscillation amplitude at different pressures. Corresponding fast Fourier transform (FFT) spectra of (b) T $\mathrm{T}_{\mathrm{d}}$ (d) TIN, and (f) $\mathrm{T}^{\prime}$ emphasize the dramatic changes in quantum oscillation frequency, due to changes in the dominant electronic orbits, as pressure tunes through the different phases. g Schematic of the topological interface network (TIN) showing the 3D mixed $T_{d}-T^{\prime}$ microstructure. The relatively weak but coherent signals are robust against pressure-induced disorder and only come from the connected interfaces between grains of $T_{d}$ and $T^{\prime}$. This TIN heterostructure can parametrically increase the number of surface channels and is a promising approach to increasing the surface-to-volume ratio of mixed-phase topological materials. The $\mathrm{SdH}$ signal is only related to cyclotron motion in the ab plane since electric current (I) and magnetic field (B) are along a and c crystallographic axis.

$\mu S R$ measurements ${ }^{18}$. The combination of pressure-sensitive structure and topology in $\mathrm{MoTe}_{2}$ makes this an exciting playground for the use of topological interfaces, with potential applications in a variety of quantum devices.

\section{METHODS}

First-principles calculations

The total energy, structure optimization under pressure, and band structure and Fermi-surface calculations were performed by Quantum Espresso $^{21}$, which is based on DFT, using a plane-wave basis set and fully relativistic all-electron projected augmented wave (PAW) potentials ${ }^{22}$. The $4 s, 4 p, 4 d$, and $5 s$ electrons of Mo and the $4 d, 5 s$, and $5 p$ electrons of Te were treated as valence. We used 0.02 Ry Methfessel-Paxton smearing with wavefunction and charge density cut-off energies of 100 Ry and $800 \mathrm{Ry}$, respectively. The exchange-correlation interactions were described by the generalized gradient approximation (GGA) with the Perdew-Burke-Ernzerhof exchange-correlation functional ${ }^{23}$. The Brillouinzone integration was performed using Monkhorst-Pack grids of special points with $16 \times 8 \times 4$ for total energy and structure optimizations and $32 \times 16 \times 8$ with tetrahedra method for electronic density of states and Fermi-surface calculations. The spin-orbit (SO) interactions and the weak inter-layer van der Waals (vdW) interactions were all included in our calculations. We used grimme- $\mathrm{d} 2^{24} \mathrm{vdW}$ correction with parameter london $-s 6=0.6$. The effect of electron correlations is included within DFT $+U$ method with $\mathrm{U}=3.0 \mathrm{eV}$ for the Mo $4 \mathrm{~d}$-states. Including electron correlation brings the calculated band structure and Fermi surface into an excellent agreement with quantum oscillation and ARPES measurements, as discussed in Supplementary Information and also found in other very recent studies ${ }^{25,26}$. Fermi-surface sheets and SdH orbits are visualized by our custom python code using Mayavi ${ }^{27}$. The quantum oscillation frequencies/orbits and their angle dependence were calculated using the skeaf $\operatorname{code}^{28}$.

\section{Crystal synthesis}

Large single crystals were grown using the Te self flux method described elsewhere ${ }^{12}$ using high purity Mo powder $(5 \mathrm{~N}$ metals basis excluding W, Alpha Aesar), and Te shot (6N, Alpha Aesar). High sample quality has been confirmed by X-ray and neutron diffraction, stoichiometry has been confirmed by wavelength dispersive spectroscopy, and the samples measured have residual resistivity ratios $>1000$.

\section{Structural measurements}

Determinations of the temperature and pressure-dependent crystal structure were made using elastic neutron scattering measurements at $14.7 \mathrm{meV}$ on the BT-4 triple-axis spectrometer at the NIST Center for Neutron Research using a collimation and filter setup of open-pg-40'-pg-spg- $40^{\prime}-120^{\prime}$, where pg refers to pyrolytic graphite. Single crystals were mounted in a steel measurement cell aligned in the $\mathrm{HOL}_{M}$ zone, and $\mathrm{He}$ was supplied as a pressure medium to maintain hydrostatic pressure conditions as described elsewhere ${ }^{12}$. The $T_{d}$ and $T^{\prime}$ phases and their volume fractions were identified from the position and intensity of (201) $M$ reflections, which both split in $2 \theta$ and shift in $\omega$ in the $\mathrm{T}^{\prime}$ phase. Rocking curves and $\omega-2 \theta$ scans were taken at each pressure and temperature. Scans along (00L) from (2 00.5$)$ to $(204.5)$ were also obtained at $0.8 \mathrm{GPa}$ in all $\mathrm{T}_{\mathrm{d}}$ or $\mathrm{T}^{\prime}$ condition, as well as in the mixed region at both 0.8 and $1 \mathrm{GPa}$ to look for possible superstructure reflections. None were observed.

\section{Transport measurements}

A nonmagnetic piston-cylinder pressure cell was used for transport measurements under pressure up to $1.8 \mathrm{GPa}$, using a 1:1 ratio of n-Pentane to 1-methyl-3-butanol as the pressure medium and superconducting temperature of lead as pressure gauge at base temperature.

All transport measurements were performed on the same MoTe $\mathrm{M}_{2}$ crystal with $110-\mu \mathrm{m}$ thickness using four-point contacts made with silver epoxy. The pressure dependence of the superconducting transition and structural 
(a) $T_{d} T^{\prime}$ Superstructure

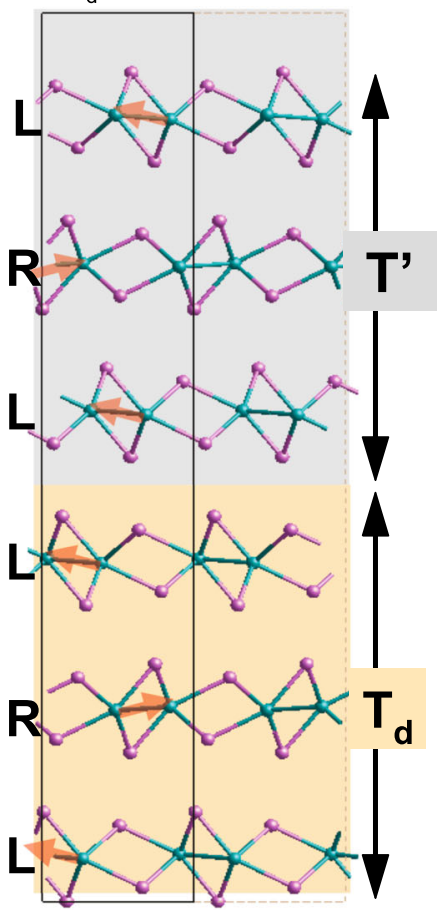

(b) $T_{d} T^{\prime}$ Slab
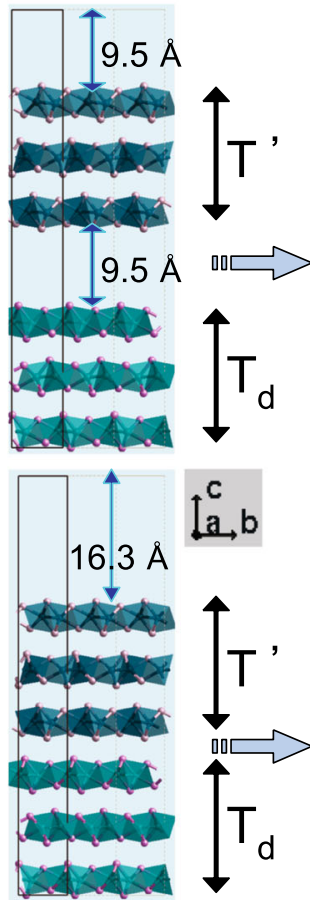

(c) Fermi surface of $\mathrm{T}_{d} \mathrm{~T}^{\prime}$ slabs
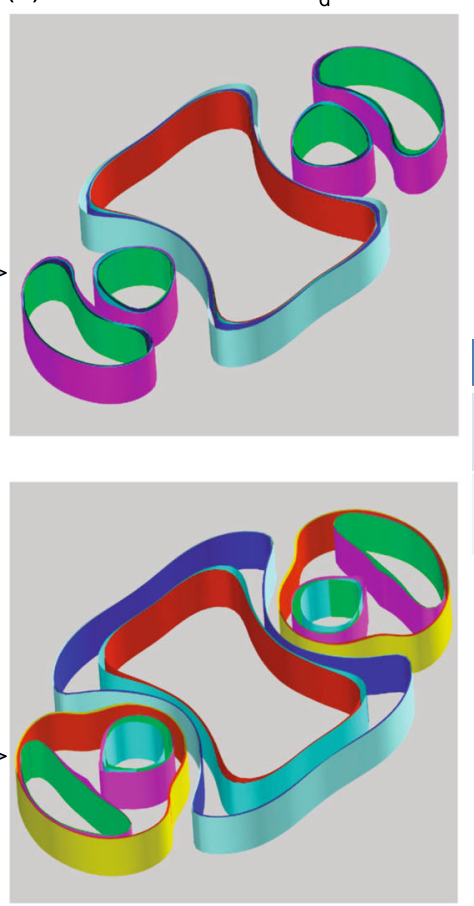

(d) Interface Femi-pockets
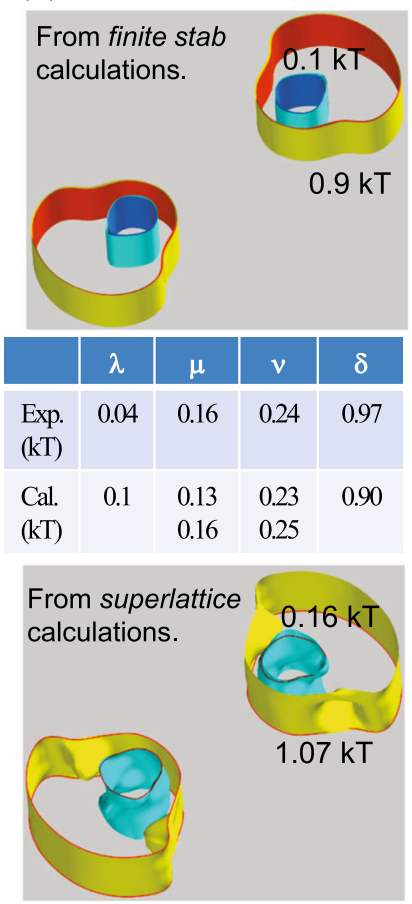

Fig. 5 The topological interface network $\mathbf{T}_{d}-\mathbf{T}^{\prime}$ superstructure model. a Six layers of $T_{d}-T^{\prime}$ periodic superstructure, consisting of three layers of $T_{d}$ and $T^{\prime}$ phases with $L-L$ interface. $\mathbf{b}$ Three layers of $T_{d}$ and $T^{\prime}$ slabs, separated (top) and joined (bottom). c Fermi surface obtained from separated (top) and joined slabs (bottom). d-top: The difference in the Fermi surfaces of the separated (c-top) and joined slabs (c-bottom), directly indicating the states due to the $T_{d}-T^{\prime}$ interface. Similarly, (d-bottom) shows the interface Fermi pockets from the periodic superstructure shown in (a). The middle panel in (d) shows the quantum oscillations from the $T_{d}-T^{\prime}$ joint slab calculations (b-bottom) compared with the experimental frequencies, which are represented as Gaussian curves with equal but arbitrary intensities.

transition $^{12}$ were determined in a Quantum Design PPMS and dilution refrigerator in the first pressure run (1 atm to $1.8 \mathrm{Gpa}$ and back to $1 \mathrm{~atm})$. The $\mathrm{SdH}$ oscillations were measured in a PPMS and dilution refrigerator (only $0.6 \mathrm{Gpa}$ ) in the second pressure run ( $1 \mathrm{~atm}$ to $1.8 \mathrm{Gpa}$ and back to 1 atm) and in an Oxford Instruments Heliox at $0.3 \mathrm{~K}$ and 6-18 $\mathrm{T}$ in a third pressure run (pressure range below $1.8 \mathrm{Gpa}$ ). The identification of commercial equipment does not imply endorsement by NIST.

\section{DATA AVAILABILITY}

Data are available from the authors upon reasonable request.

Received: 5 March 2020; Accepted: 14 August 2020;

Published online: 07 September 2020

\section{REFERENCES}

1. Kitaev, A. Y. Fault-tolerant quantum computation by anyons. Ann. Phys. 303, 2-30 (2003).

2. Nayak, C. et al. Non-Abelian anyons and topological quantum computation. Rev. Mod. Phys. 80, 1083-1159 (2008).

3. Qi, Y. et al. Superconductivity in Weyl semimetal candidate $\mathrm{MoTe}_{2}$. Nat. Commun. 7, 11038 (2016).

4. Qian, X., Liu, J., Fu, L. \& Li, J. Quantum spin Hall effect in two-dimensional transition metal dichalcogenides. Science 346, 1344-1347 (2014).

5. Hsu, Y.-T., Vaezi, A., Fischer, M. H. \& Kim, E.-A. Topological superconductivity in monolayer transition metal dichalcogenides. Nat. Commun. 8, 14985 (2017).

6. Li, Y. et al. Nontrivial superconductivity in topological MoTe $\mathrm{M}_{2-x} \mathrm{~S}_{x}$ crystals. Proc. Natl Acad. Sci. USA 115, 9503-9508 (2018).

7. Soluyanov, A. A. et al. Type-II Weyl semimetals. Nature 527, 495-498 (2015).

8. Deng, K. et al. Experimental observation of topological Fermi arcs in type-Il Weyl semimetal $\mathrm{MoTe}_{2}$. Nat. Phys. 7, 1105-1110 (2016).
9. Huang, L. et al. Spectroscopic evidence for type II Weyl semimetallic state in $\mathrm{MoTe}_{2}$. Nat. Mater. 15, 1155-1160 (2016).

10. Jiang, J. et al. Signature of type-ll Weyl semimetal phase in $\mathrm{MoTe}_{2}$. Nat. Commun. 8, 13973 (2017).

11. Wang, Z. et al. Higher-order topology, monopole nodal lines, and the origin of large Fermi arcs in transition metal dichalcogenides $\mathrm{XTe}_{2}(\mathrm{X}=\mathrm{Mo}, \mathrm{W})$. Phys. Rev. Lett. 123, 186401 (2019)

12. Heikes, $C$. et al. Mechanical control of crystal symmetry and superconductivity in Weyl semimetal MoTe 2 . Phys. Rev. M. 2, 074202 (2018).

13. Crepaldi, A. et al. Persistence of a surface state arc in the topologically trivial phase of $\mathrm{MoTe}_{2}$. Phys. Rev. B 95, 041408 (2017).

14. Rhodes, D. et al. Bulk Fermi surface of the Weyl type-Il semimetallic candidate $\gamma$ -MoTe 2 . Phys. Rev. B 96, 165134 (2017).

15. Kienzle, P. Bumps: curve fitting and uncertainty analysis (Version 0.7.10). Preprint at http://readthedocs.org/projects/bumps/downloads/pdf/latest/ (2018).

16. Murakawa, H. et al. Detection of Berryas phase in a bulk Rashba semiconductor. Science 342, 1490-1493 (2013).

17. Luo, X. et al. $\mathrm{T}_{d}-\mathrm{MoTe}_{2}$ : a possible topological superconductor. Appl. Phys. Lett. 109, 102601 (2016)

18. Guguchia, Z. et al. Signatures of the topological $s^{+-}$superconducting order parameter in the type-II Weyl semimetal $T_{d}-\mathrm{MoTe}_{2}$. Nat. Commun. 8, 1082 (2017).

19. Huang, F.-T. et al. Polar and phase domain walls with conducting interfacial states in a Weyl semimetal MoTe 2 . Nat. Commun. 10, 4211 (2019).

20. $\mathrm{Hu}, \mathrm{Y}$. J. et al. Angular dependence of the upper critical field in the high-pressure $1 T^{\prime}$ phase of $\mathrm{MoTe}_{2}$. Phys. Rev. M 3, 034210 (2019).

21. Giannozzi, P. et al. Quantum espresso: a modular and open-source software project for quantum simulations of materials. Matter 21, 395502 (2009).

22. Kresse, G. \& Joubert, D. From ultrasoft pseudopotentials to the projector augmented-wave method. Phys. Rev. B 59, 1758-1775 (1999).

23. Perdew, J. P., Burke, K. \& Ernzerhof, M. Generalized gradient approximation made simple. Phys. Rev. Lett. 77, 3865-3868 (1996).

24. Grimme, S. Semiempirical GGA-type density functional constructed with a longrange dispersion correction. J. Comp. Chem. 27, 1787-1799 (2006),

25. $\mathrm{Xu}, \mathrm{N}$. et al. Evidence of Coulomb-interaction-induced Lifshitz transition and robust hybrid Weyl semimetal in $T_{d}-M_{0} e_{2}$. Phys. Rev. Lett. 121, 136401 (2018). 
26. Aryal, N. \& Manousakis, E. Importance of electron correlations in understanding the photo-electron spectroscopy and the Weyl character of $\mathrm{MoTe}_{2}$. Phys. Rev. B 99, 035123 (2019).

27. Ramachandran, P. \& Varoquaux, M. 3d visualization of scientific data. IEEE Comput. Sci. Eng. 13, 40-51 (2011).

28. Rourke, P. M. C. \& Julian, S. R. Numerical extraction of de Haas-van Alphen frequencies from calculated band energies. Comp. Phys. Commun. 183, 324-332 (2012).

\section{ACKNOWLEDGEMENTS}

We thank Jay D. Sau, Paul Kienzle, and Daniel J. Campbell for helpful discussions. We specially appreciate John Singleton for the thorough discussion on the analysis of the $\mathrm{g}$ factor and Berry phase in Lifshitz-Kosevich (LK) formula. The computational work was performed using the NIST Enki high-performance computer cluster. This work utilized facilities supported in part by the National Science Foundation under Agreement No. DMR-0454672 and NCNR/UMD Cooperative Agreement 70NANB15H261. Experimental measurements at the University of Maryland were supported by DOE through Grant No. DE-SC-0019154, and materials synthesis by the Gordon and Betty Moore Foundation's EPiQS Initiative through Grant No. GBMF4419. Portions of the experimental research were supported by NIST.

\section{AUTHOR CONTRIBUTIONS}

I.L., C.E., and T.M. performed transport measurements. C.H. and W.R. measured neutron scattering. N.P.B., C.H., and S.R. synthesized materials. T.Y. performed electron band structure calculations and theoretical analysis. I.L., T.Y., C.H., and N.P.B. wrote the paper with contributions from all authors.

\section{COMPETING INTERESTS}

The authors declare no competing interests.

\section{ADDITIONAL INFORMATION}

Supplementary information is available for this paper at https://doi.org/10.1038/ s41535-020-00264-8.

Correspondence and requests for materials should be addressed to IL.L. or N.P.B.

Reprints and permission information is available at http://www.nature.com/ reprints

Publisher's note Springer Nature remains neutral with regard to jurisdictional claims in published maps and institutional affiliations.

Open Access This article is licensed under a Creative Commons Attribution 4.0 International License, which permits use, sharing, adaptation, distribution and reproduction in any medium or format, as long as you give appropriate credit to the original author(s) and the source, provide a link to the Creative Commons license, and indicate if changes were made. The images or other third party material in this article are included in the article's Creative Commons license, unless indicated otherwise in a credit line to the material. If material is not included in the article's Creative Commons license and your intended use is not permitted by statutory regulation or exceeds the permitted use, you will need to obtain permission directly from the copyright holder. To view a copy of this license, visit http://creativecommons. org/licenses/by/4.0/.

This is a U.S. government work and not under copyright protection in the U.S.; foreign copyright protection may apply 2020 